\title{
A simple pregnancy-related kidney disease screening method: random proteinuria testing
}

\author{
Won Kim
}

Department of Internal Medicine, Chonbuk National University Medical School, Jeonju, Korea

Received: October 9, 2017

Accepted: October 12, 2017

\section{Correspondence to}

Won Kim, M.D.

Department of Internal Medicine, Chonbuk National University Medical School, 20 Geon-

ji-ro, Deokjin-gu, Jeonju 54907,

Korea

Tel: $+82-63-250-1651$

Fax: +82-63-254-1609

E-mail: kwon@jbnu.ac.kr

\section{See Article on Page 1062-1068}

Many kidney-related pathologic conditions can affect the outcome of pregnancy, including chronic kidney disease, preeclampsia, eclampsia, underlying hypertension, diabetes mellitus, and glomerular disease. In addition, there are pregnancy-related kidney diseases whose course may vary during pregnancy. The diagnosis of these diseases in their early stages is critical to decreasing pregnancy-associated morbidity or mortality. In addition, several glomerular diseases may develop or be aggravated during pregnancy, including lupus nephritis, antiphospholipid antibody syndrome, diabetic nephropathy, minimal change disease, membranous nephropathy, immunoglobulin A nephropathy, vasculitis syndrome, atypical hemolytic uremic syndrome, and eclampsia/preeclampsia [1].

Proteinuria is already an established biomarker, similar to serum creatinine and albuminuria, that yields diagnostic information in non-pregnancy-related kidney diseases, including chronic kidney disease, diabetic nephropathy, hypertensive nephropathy, and some glomerulopathies [2]. Their worsening or development during pregnancy is often accompanied by proteinuria. While these and the above-described conditions suggest that proteinuria should be assessed during pregnancy, either as a diagnostic indicator or in the monitoring of kidney disease, whether proteinuria is a marker for the detection of a relevant subset of kidney diseases occurring during pregnancy remains unclear.

There are several hurdles to the use of proteinuria as a marker of kidney disease during pregnancy. The upper limit of 24hour urine protein in non-pregnant women is $150 \mathrm{mg} /$ day. Physiological changes related to pregnancy may increase proteinuria by up to $300 \mathrm{mg} /$ day due to the increased glomerular filtration rate and greater permeability of the glomerular basement membrane [3]. Therefore, women with a normal pregnancy without complicated renal disease may still have an increased urinary protein level.

Methods for testing for proteinuria can also pose challenges. Random proteinuria testing is simple and easy to perform, but the results can vary depending on the urine concentration and they have the disadvantage of being time-variant. Also, 24-hour protein data cannot be collected in a single test. Determination of the urinary protein-to-creatinine ratio has the advantage of providing a measurement of 24 -hour protein values, but it requires special equipment and can be time consuming and thus not useful in all settings.

Proteinuria may occur or worsen in many renal diseases, but is absent in others. In one study, $19 \%$ of eclampsia 
patients did not have proteinuria [4], nor is proteinuria an essential diagnostic component of preeclampsia [5].

Although random proteinuria testing has its limitations, as noted above, it has the advantage of being a simple method that can be used by physicians who are not kidney specialists. Thus, a simple and rapid screening test is needed to detect kidney disease in pregnant women. Random urine tests to measure proteinuria may be informative, albeit not conclusive in the absence of proteinuria.

Bae et al. [6] assessed the effects of random urine proteinuria during pregnancy on maternal and fetal outcomes. In their retrospective case-control study, the incidences of preeclampsia, preterm labor, premature membrane rupture, and intrauterine growth restriction were significantly higher in women with random proteinuria. However, Yamada et al. [7] recently reported that proteinuria measured via the urine dipstick test may overestimate the risk of significant proteinuria. Thus, controversy remains regarding the association between random urine protein and kidney diseases during pregnancy. Nonetheless, considering the potential utility of random urine protein screening for kidney disease during pregnancy and the convenience of the method, it may be informative. In their large study, Bae et al. [6] suggested random screening for proteinuria as a means of evaluating the risk of preeclampsia, preterm labor, premature membrane rupture, and intrauterine growth restriction.

The authors also found a link between random urine protein and the incidence of hematuria in pregnant women. The incidence of proteinuria (19.0\%) was significantly higher than that of hematuria (8.8\%). Based on their results, they advocated the use of hematuria and proteinuria as potential markers associated with pregnancy-related complications. Recently, Shahraki et al. [8] reported that hematuria was associated with abortion and neonatal death, but not with other pregnancy-related complications. Thus, additional studies are needed to validate this approach.

Decreased renal function negatively affects pregnancy outcomes, and pregnancy can impact chronic kidney disease. Diabetic nephropathy may also have a role in renal disease during pregnancy. The frequency of chronic kidney disease (1.0\%) and diabetes mellitus (1.4\%) was low in the study of Bae et al. [6]. Thus, the effect of these two diseases on proteinuria in pregnancy may be relatively low. An association between symptomatic urinary tract dilata- tion and proteinuria in pregnancy has also been described [9]. Accordingly, dilatation of the urinary tract should be checked during the differential diagnosis of proteinuria in pregnancy.

In summary, random urine proteinuria testing may be a useful screening tool in pregnant women in terms of both maternal and fetal outcomes.

\section{Conflict of interest}

No potential conflict of interest relevant to this article was reported.

\section{REFERENCES}

1. Smyth A, Radovic M, Garovic VD. Women, kidney disease, and pregnancy. Adv Chronic Kidney Dis 2013;20:402-410.

2. Gentile G, Remuzzi G. Novel biomarkers for renal diseases? None for the moment (but one). J Biomol Screen 2016;21:655-670.

3. Higby K, Suiter CR, Phelps JY, Siler-Khodr T, Langer O. Normal values of urinary albumin and total protein excretion during pregnancy. Am J Obstet Gynecol 1994;171:984989.

4. Sibai BM. Eclampsia. VI. Maternal-perinatal outcome in 254 consecutive cases. Am J Obstet Gynecol 1990;163:10491054 .

5. Thornton CE, Makris A, Ogle RF, Tooher JM, Hennessy A. Role of proteinuria in defining pre-eclampsia: clinical outcomes for women and babies. Clin Exp Pharmacol Physiol 2010;37:466-470.

6. Bae EH, Kim JW, Choi HS, Ma SK, Kim SW. Impact of random urine proteinuria on maternal and fetal outcomes of pregnancy: a retrospective case-control study. Korean J Intern Med 2017;32:1062-1068.

7. Yamada T, Kojima T, Akaishi R, et al. Problems in methods for the detection of significant proteinuria in pregnancy. $J$ Obstet Gynaecol Res 2014;40:161-166.

8. Shahraki AD, Bardeh ME, Najarzadegan MR. Investigation of the relationship between idiopathic microscopic hematuria (in the first and second trimesters) and major adverse outcomes of pregnancy. Adv Biomed Res 2016;5:186.

9. Piccoli GB, Attini R, Parisi S, et al. Excessive urinary tract dilatation and proteinuria in pregnancy: a common and overlooked association? BMC Nephrol 2013;14:52. 